Original Paper http://ajol.info/index.php/ijbcs http://indexmedicus.afro.who.int

\title{
Corrélats écologiques de dégâts d'éléphants et efficacités des techniques traditionnelles de refoulement : cas de la forêt classée de Niangoloko, Burkina Faso
}

\author{
Djidama SIRIMA $^{1 *}$, Idrissa KABORE ${ }^{1}$, M. Emmanuel HEMA ${ }^{1,2}$ et \\ Boureima Gustave KABRE ${ }^{1}$ \\ ${ }^{1}$ Laboratoire de Biologie et Ecologie Animales, Université Joseph KI-ZERBO, 09 B.P. 848 Ouagadougou \\ 09, Ouagadougou, Burkina Faso. \\ ${ }^{2}$ Université de Dédougou, UFR/Sciences Appliquées et Technologiques, Dédougou, Burkina Faso. \\ *Corresponding author; E-mail: sirima_djidama@yahoo.fr; Tel: (+226) 72361426
}

\section{REMERCIEMENTS}

Cette étude a été financée par IFS (Fondation Internationale pour la Science).

\section{RÉSUMÉ}

Présents dans la forêt depuis 2011, les éléphants ont entrainé un grand changement dans le quotidien des producteurs autours de la forêt classée de Niangoloko (FCN). Les pertes des cultures de plus en plus croissantes ont entrainé une interaction négative entre les Hommes et les éléphants. Afin d'identifier d'une part, les corrélats écologiques qui expliquent au mieux l'ampleur des dégâts dans les champs et d'autre part, discuter de l'efficacité des techniques de refoulement d'éléphant utilisées par les producteurs ; nous avons conduit une étude durant trois saisons agricoles (2016-2018) autours de la FCN. À cet effet, nous avons réalisé un suivi systématique des dégâts d'éléphants et des techniques de refoulement utilisées dans les champs situés dans un rayon de $10 \mathrm{~km}$ autour de la forêt de juin à décembre. Les résultats ont montré qu'un total cumulé de 2496,4 ha de champs ont été cultivés et un total de dégâts d'éléphants de 1321 ha soit $53 \%$ de la superficie totale cultivée. Au total 664 producteurs ont été victimes et 14 techniques de refoulement (TR) ont été recensées. Les résultats du test de Kruskal-Wallis entre le taux de dégâts (TD) et les TR montrent que le TD était significativement différente $(\mathrm{p}=0,000 ; \mathrm{n}=664)$ d'une TR à une autre. Par ailleurs, les résultats du GLM (General linear model) montrent que l'altitude influençait positivement le TD $(\mathrm{p}=0,025)$. Les résultats de cette étude pourraient être mis au profit des producteurs pour une réduction des conflits Hommes-éléphants au Burkina Faso.

(C) 2020 International Formulae Group. All rights reserved.

Mots clés : Conflit, éléphants, techniques de refoulement, altitude, Niangoloko, Burkina Faso.

\section{Ecological correlates of elephant damage and the effectiveness of traditional repression techniques: The case of the Niangoloko classified forest, Burkina Faso}

\begin{abstract}
Present in the forest since 2011, the elephants have brought a big change in the daily life of famers around the Niangoloko classified forest of (NCF). The losses of growing crops have resulted in a negative interaction between humans and elephants. In order to identify, on the one hand, the ecological correlates which best explain the extent of the damage in the fields, on the other hand, to discuss the effectiveness of the elephant repression techniques used by famers; we conducted a study during three agricultural seasons (2016-2018)
\end{abstract}


around the NCF. To this end, we systematically monitored elephant damage and repression techniques used from farms located within a $10 \mathrm{~km}$ radius of the forest from june to december. The results showed that a cumulative total of 2496.4 ha of farms were cultivated and a total of 1321 ha or $53 \%$ of cultivated area are damaged by elephants. A total of 664 famers were victims and 14 repression techniques (RT) were identified. The results of the Kruskal-Wallis test between the damage rate (DR) and the RT show that the DR was significantly different $(\mathrm{p}=0.000 ; \mathrm{n}=664)$ from one TR to another. In addition, the results of the GLM (General linear model) show that the altitude positively influenced the DR $(\mathrm{p}=0.025)$. The results of this study could be used for the benefit of famers to reduce human-elephant conflicts in Burkina Faso.

(C) 2020 International Formulae Group. All rights reserved.

Keywords: Conflict, elephants, pushback techniques, altitude, Niangoloko, Burkina Faso.

\section{INTRODUCTION}

L'agriculture est l'activité principale qui menace la survie des aires protégées en Afrique (Hedges et Gunaryadi, 2010). L'occupation des terres autour des aires de conservation engendre des conflits entre les éléphants et les producteurs locaux. Les causes de ces conflits sont attribuables aux modes d'utilisation des terres, à la croissance démographique et aussi à la pauvreté (Parker et al., 2007; MacKinnon et al., 2015). En effet, ces conflits Homme-éléphant (CHE) constituent un problème préoccupant dans les zones rurales et peuvent entrainer des effets néfastes aussi bien sur les populations humaines et que sur les éléphants (MacKinnon et al., 2015). Ces conflits sont encore plus perceptibles dans les pays à revenu faible avec une économie basée sur l'agriculture.

Au Burkina Faso, les CHE dans les zones à forte densité d'éléphants ont été décrits dans les travaux de Hien (2001), Nakandé et al. (2007) et Hema et al. (2018); et Les activités agricoles autour des aires ne cessent de s'intensifier, posant un véritable problème de gestion durable de la faune. Les éléphants particulièrement causent d'énormes dégâts aux cultures des communautés rurales vivant autour de la forêt classée de Niangoloko $(\mathrm{FCN})$. Sur le terrain, plusieurs moyens et techniques sont mis en œuvre par les producteurs pour dissuader les éléphants. Cependant l'efficacité de ces techniques reste discutable et les résultats souvent mitigés. La présente étude qui se veut le premier diagnostic des approches locales autour de la FCN contribuera à la consolidation des connaissances pour une meilleure gestion des ressources naturelles au Burkina Faso. Spécifiquement, nous allons analyser l'ampleur des dégâts d'éléphants sur les spéculations, discuter de l'efficacité des techniques de refoulements utilisées par les producteurs et identifier les corrélats qui ont un effet significatif sur l'ampleur de dégât autours de la forêt classée de Niangoloko. Les résultats de cette étude contribueront à une meilleure compréhension du phénomène et surtout ils contribueront à mieux conserver les petites populations d'éléphants vivant dans la savane et surtout dans les milieux fortement anthropisés.

\section{MATÉRIEL ET MÉTHODES Site d'étude}

La forêt classée de Niangoloko (FCN), s'étend sur une superficie de 7295,38 hectares. Géographiquement, elle est comprise entre les longitudes $4^{\circ} 52^{\prime}$ et $4^{\circ} 58^{\prime} \mathrm{W}$ et entre les latitudes $10^{\circ} 10^{\prime}$ et $10^{\circ} 16^{\prime} \mathrm{N}$ (Figure 1). Les principales localités riveraines de la forêt classée sont : deux secteurs de la commune de Niangoloko (Diakora: secteur 8) et (Djole: secteur 9) au Nord, le village de Yindéré au Sud-Ouest, le village de Timpereba au Sud et les villages de Moutieridougou, de Diakora et Diole au Nord-Est. Le Village de Kakoumana constitue une enclave dans la forêt classée (Ouoba et al., 2006). Le secteur primaire à savoir l'agriculture, l'élevage, la pêche, occupe $86,9 \%$ de la population dans cette région (INSD, 2017). La FCN est située dans le secteur soudanien méridional, plus précisément dans le district de la Comoé (Guinko, 1984) avec des températures moyennes variant entre $25^{\circ}-31^{\circ} \mathrm{C}$. $\mathrm{La}$ végétation de la forêt classée est une mosaïque de forêt galeries et de savanes boisées et arbustives (Ouoba et al., 2006). 
Méthodes de collecte de données

Cette étude a été réalisée entre juin 2016 et novembre à 2018. Une enquête ethnozoologique a été utilisée pour l'enregistrement des données. L'approche utilisée sur le terrain est identique à celle que nous avons utilisée autour de la Forêt Classée et Ranch de Gibier de Nazinga (Hema et al., 2018). Dans le cas de la FCN, six (6) villages situés dans un rayon de $10 \mathrm{~km}$ de la forêt ont été concernées par cette étude. Des visites de terrains ont été organisées pour confirmer les résultats des enquêtes. Les données collectées ont concernées, les spéculations endommagées, la superficie cultivée (SC), la superficie endommagée (SD), la frequence de visite des éléphants dans les champs durant les saisons agricoles et les differentes techniques de refoulement utilisées.

\section{Nomenclature des techniques et methodes de roulements utilisées dans les champs}

Nous avons appelé «technique de refoulement » tout mecanisme (protocole) de refoulement mis en œuvre à la fois par un producteur dans un même champ donné; et nous avons appelé «méthode de refoulement » chaque moyen (ou outil) different de refoulement utilisé pour dissuader les éléphants dans les champs. Ainsi une «technique de refoulement » utilisée dans un champ est composée de une ou plusieurs «méthode de refoulement ». En d'autres terme, l'usage d'une «méthode de refoulement » en solo ou en combiné dans un champ donné à été considéré comme la «technique de refoulement » de ce champ.

\section{Estimations des taux de dégâts}

Pour chaque type de culture, les taux de dégâts de cultures des éléphants (TD) ont été calculées par la formule suivante :

$$
\mathrm{TD}=\mathrm{SD} * 100 / \mathrm{SC}
$$

où $\mathrm{SD}$ represente la superficie détruite après la visite des éléphants et SC la superficie totale de champ cultivée.

La superficie cultivée a été estimée en parcourant les limites de chaque champ à l'aide d'un GPS ; tandis que la superficie de champ détruite a été calculée sur la base du rendement moyen à l'hectare de chaque spéculation au niveau de la zone d'étude (FAO et AFRISTAT, 2000; MAHRH, 2008) et aussi sur la base de la méthode utilisant la déclaration du producteur d'un interrogatoire auprès de chaque producteur victime tel que decrite dans le document de la FAO et AFRISTAT (2000).

$$
\mathrm{SD}=(\mathrm{Pa}-\mathrm{Pr}) / \mathrm{Re}
$$

où $\mathrm{Pa}$ represente la production attendue en tonne, $\mathrm{Pr}$ la recolte en tonne et $\mathrm{Re}$ le rendement à l'hectare de la spéculation concernée.

\section{Analyses statistiques \\ Comparaison des moyennes de taux de dégât autour de la FCN}

La normalité de la variable dépendante, des effets aléatoires et des erreurs ont été testées à l'aide test de Shapiro-Wilk $(\mathrm{p}<5 \%)$. La comparaison des moyennes de taux de dégât entre les années, entre les types de spéculation cultivées et aussi entre les techniques de refoulement utilisées autour de la FCN a été réalisée en faisant le test de Kruskal-Wallis

Relation taux de dégât et nombre de visite d'éléphants dans le champ

Le test de corrélation de Pearson a été utilisé pour analyser la relation entre les taux de dégât et le nombre de visite d'éléphants dans le champ.

Analyse de l'influence des variables explicatives sur le taux de dégât

Le Modèle Linéaire généralisé (GLM, Hosmer et Lemeshow, 2000) a été utilisé pour mesurer les effets des variables indépendantes sur le taux de dégâts. Dans chaque analyse, les taux de dégât de culture ont été utilisés comme variables dépendantes tandis que les variables résumées dans le Tableau 1 ont été utilisés comme variables explicatives. Le GLM a également été utilisé pour analyser l'effet des techniques (Tableau 1) sur le taux de dégât. La méthode d'analyse utilisant la procédure pas à pas "stepwise" ascendante a été employée et les variables ont été exclues quand elles n'avaient pas de corrélation significative avec la variable dépendante ( $\chi^{2}$ de Wald; $\left.p>0,05\right)$. Les variables retenues ont été utilisées dans le calcul du modèle. Les modèles retenus ont été calculés en utilisant la procédure du meilleur sous-ensemble. Les données ont été analysées sous le logiciel SPSS version 21. 


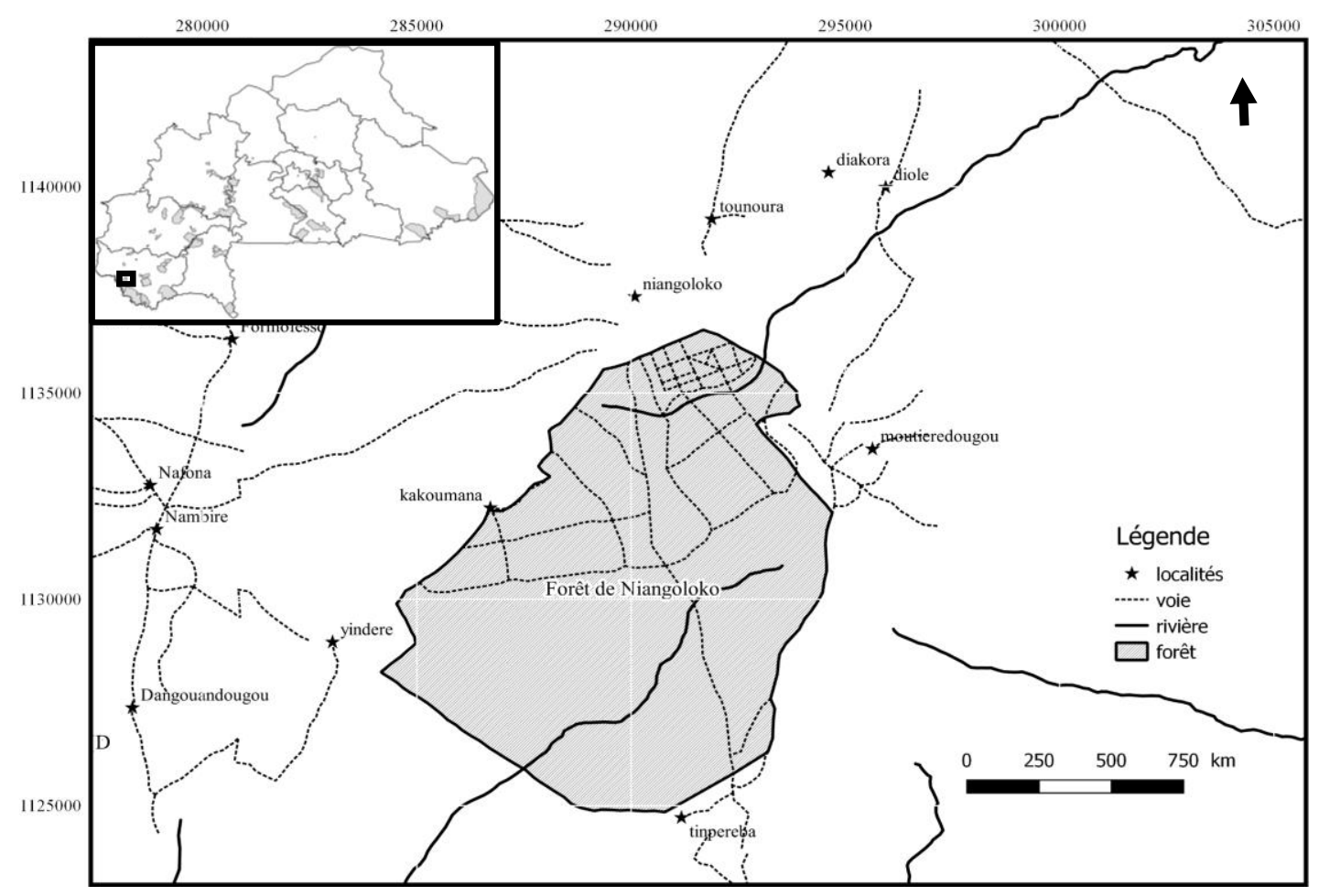

Figure : Présentation de la zone d'étude.

Tableau 1: Liste des variables indépendantes et les scores pour celles qui sont nominales lors de l'analyse statistiques

\begin{tabular}{ll}
\hline Variables & Signification \\
\hline Spéculation & Les types de spéculations endommagés \\
\hline $\begin{array}{l}\text { Techniques } \\
\text { Refoulement } \\
(\mathrm{TR})\end{array}$ & $\begin{array}{l}\text { Les techniques de refoulement utilisées par les producteurs autours de la } \\
\text { FCN }\end{array}$ \\
\hline Champ & Distance séparant deux champs voisins endommagés par les éléphants \\
\hline Rivière & $\begin{array}{l}\text { Distance séparant un champ endommagé par les éléphants de la rivière la } \\
\text { plus proche }\end{array}$ \\
\hline Voie & $\begin{array}{l}\text { Distance séparant un champ endommagé par les éléphants de la de voie } \\
\text { de communication la plus proche }\end{array}$ \\
\hline Village & Distance séparant un champ du village le plus proche \\
\hline Bas-fond & $\begin{array}{l}\text { Distance séparant un champ endommagé par les éléphants du bas-fond le } \\
\text { plus proche }\end{array}$ \\
\hline Forêt & $\begin{array}{l}\text { Distance séparant un champ endommagé par les éléphants de la limite de } \\
\text { la FCN }\end{array}$ \\
\hline Altitude & $\begin{array}{l}\text { Il s'agit de la position du champ par rapport au niveau de la mer, donnée } \\
\text { par le GPS }\end{array}$ \\
\hline
\end{tabular}




\section{RÉSULTATS}

Durant les trois saisons agricoles, nous avons enregistré 3692 incidents sur un total de 664 champs inventoriés, soit une moyenne de 1304 incidents par an avec un taux de dégât moyen de $54 \%$. Les spéculations ayant enregistrées les plus grosses pertes étaient le riz (Oryza sp.) (86\% des superficies cultivées), suivi du voandzou (62\% des superficies cultivées) (Vigna subterranea (L.) Verdc.) et du Sorgho (62\% des superficies cultivées) (Figure 2). Environ la moitié des champs de maïs (Zea mays L.) et sésame (Sesamum indicum L.) étaient endommagés.

Autour de la FCN, la superficie totale cultivée de 2016 à 2018 est de 2496,4 ha; $45,71 \%$ de cette superficie a été consacrée à la culture du maïs, $22,91 \%$ à la culture du coton, $12,39 \%$ à la culture d'arachide, $11,33 \%$ à la riziculture, $4,75 \%$ à la culture du sésame, $2 \%$ à la culture du sorgho, $0,77 \%$ à la culture du voandzou et enfin $0,15 \%$ à la culture du niébé (Figure 3).

\section{Technique de refoulement}

Autours de la FCN, 14 techniques de refoulement ont été utilisées par les producteurs afin de réduire les dommages causés par les éléphants aux cultures (Tableau 2). Il y avait aussi des champs où aucune technique n'était employée.

Les pertes moyennes engendrées par l'utilisation des techniques se situent entre $21 \%$ et $82 \%$. La perte la plus importante $82 \%$ a été obtenue dans les champs où le bruit $\mathrm{Br}$ était utilisé tandis que la perte la plus faible $21 \%$ a été obtenue dans les champs ayant utilisé la technique Lu-G (Figure 4).

Autour de la FCN, les champs de maïs ont été les plus nombreux, soit 153, et ils ont reçu le plus grand nombre de techniques, soit 11 techniques sur les 14 techniques totalisées; il est suivi du coton; 8 techniques ont été utilisées dans les 84 champs de coton. Quatre et trois techniques de refoulement ont respectivement été utilisées dans les champs de sorgho qui totalisait 19 champs et le niébé 4 champs (Figure 5).

Les résultats du test de Kruskal-Wallis entre le taux de dégât et les techniques de refoulement montrent que le taux de dégât était significativement différent $\quad(n=664$; $\mathrm{p}=0,000)$ d'une technique à une autre. Le même test révèle aussi que le taux de dégât était significativement différent $\quad(\mathrm{n}=664$; $\mathrm{p}=0,000)$ d'un type de spéculation à un autre (Tableau 3). Mais les résultats du test de Kruskal-Wallis montrent que d'une année à l'autre, le taux de dégât n'était pas significativement différent $(n=664 ; p=0,655)$ (Tableau 3). Les résultats du test de KruskalWallis montrent aussi qu'au niveau des techniques de refoulement $\mathrm{Br}-\mathrm{G}$ et $\mathrm{Ba}-\mathrm{Lu}-\mathrm{Br}$ $\mathrm{G}$, le taux de dégât était statistiquement différent en fonctions des années (Tableau 4).

De 2016 à 2018, parmi les 14 techniques, 9 techniques ont figurées sur la liste durant les trois années ; une technique a été abandonnée en 2017 et 2018 ; il s'agit de Ba-G dont le taux de dégât était de $33 \%$ et 3 techniques se sont ajoutées en 2017; il s'agit de Ba, Ba-Lu et Lu-G. En 2018, une seule technique s'est ajoutée, il s'agit de Ba-Lu-G et une technique a été abandonnée, il s'agit de Lu-G dont le taux de dégât moyen en 2017 était de $21 \%$ (Tableau 4).

Le nombre de champs ayant utilisé une technique donnée a connu une légère variation de 2016 à 2018. Dans l'ensemble, 5\% des champs étaient sans protection, $67 \%$ des champs endommagés n'étaient pas surveillés mais les autres techniques de protection de culture étaient utilisées, $12 \%$ des champs étaient protégés sans usage des torches, $83 \%$ des champs étaient protégés sans usage de la barrière et $8 \%$ des champs étaient protégés sans usage du bruit.

Les techniques suivantes ont connu une augmentation en terme d'utilisation dans les champs par les producteurs; il s'agit des techniques Lu-Br-G et Br, (Figure 6).

Les techniques qui ont une baisse dans leur utilisation par les producteurs sont particulièrement $\mathrm{Lu}$, Ba-Lu-Br-G et $\mathrm{Lu}-\mathrm{Br}$, (Figure 6). Certaines techniques qui n'étaient pas connues des producteurs en 2016 ont été utilisées en 2017 et 2018 ; il s'agit de la technique Ba-Lu (Figure 6). Par contre la technique Ba-G a été utilisée dans deux champs uniquement en 2016 (Figure 6). 


\section{Relation entre le taux de dégât et le nombre d'incident}

Les résultats du test de Corrélation de Pearson entre le taux de dégât et le nombre d'incident révèle que $\mathrm{R}$ vaut $12,3 \%$; $\mathrm{p}=0,001$ et $\mathrm{n}=664$ (Tableau 5).
Effet des variables explicatives sur le taux de dégât autour de la forêt classée de Niangoloko

Parmi les 9 variables explicatives retenues pour le test GLM, seul l'altitude a un effet significatif positif sur le taux de dégât. Cela veut dire que plus l'altitude augmente, le taux de dégât augmente aussi (Tableau 6).

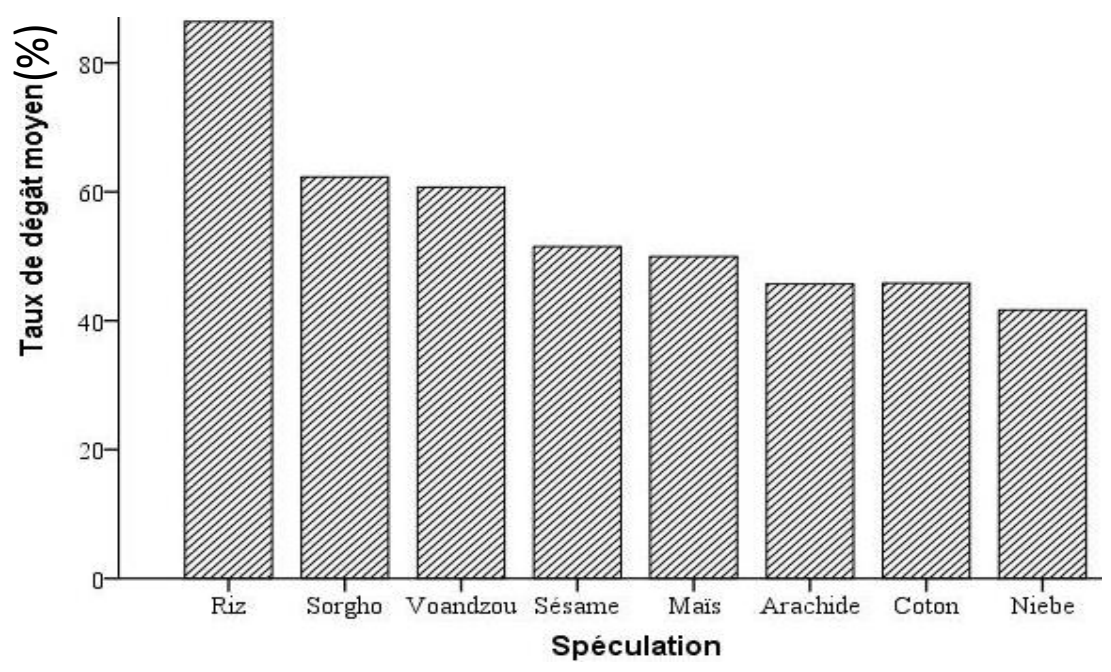

Figure 2: Taux de dégât moyen enregistré autour de la forêt classée de Niangoloko durant les trois saisons agricoles (2016 à 2018).

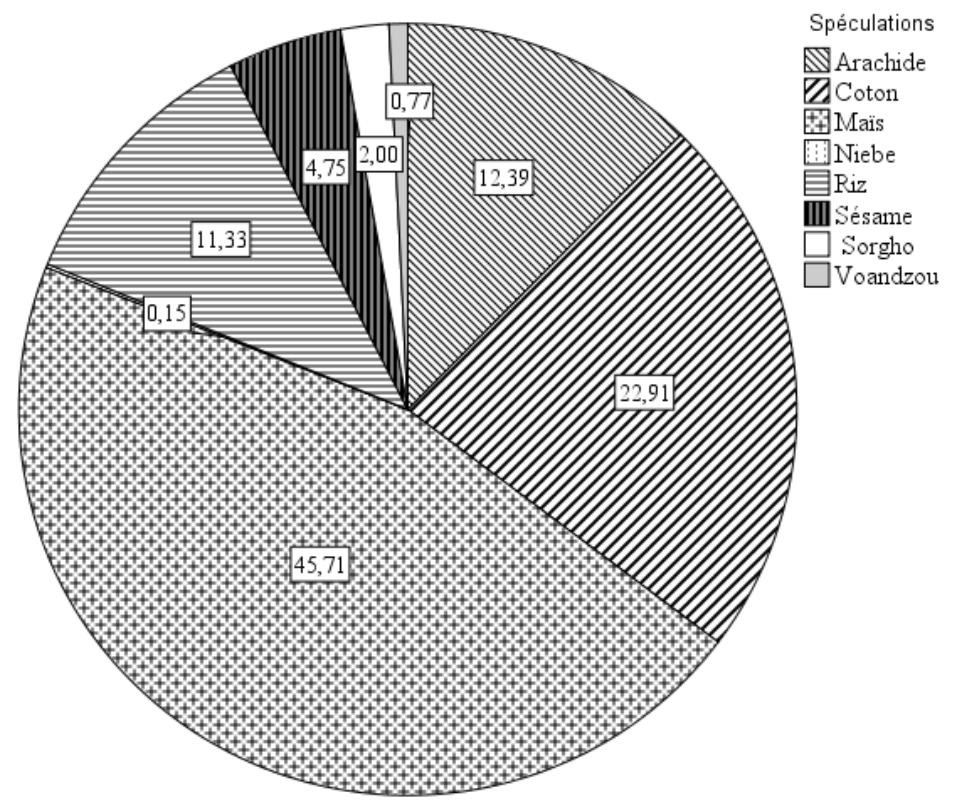

Figure 3: Répartition en pourcentage de la superficie cultivée par spéculation autour de la FCN de 2016 à 2018. 
Tableau 2: Inventaire des différents types de méthodes et les différentes techniques de refoulement utilisés autour de la forêt classée de Niangoloko.

\begin{tabular}{ll}
\hline \multicolumn{1}{l}{$\begin{array}{l}\text { Techniques de refoulement } \\
\text { Types de méthodes et techniques de refoulement en solo }\end{array}$} & Code \\
\hline Barrière & $\mathrm{Ba}$ \\
\hline Bruit & $\mathrm{Br}$ \\
\hline Gardiennage & $\mathrm{G}$ \\
\hline Torches & $\mathrm{Lu}$ \\
\hline Aucune technique & $\mathrm{Auc}$ \\
\hline \multicolumn{1}{c}{ Techniques de refoulement utilisées en combinées } \\
\hline «Barrière + Gardiennage» \\
\hline «Bruit + Gardiennage» \\
\hline «Torches + Bruit» & $\mathrm{Ba}-\mathrm{G}$ \\
\hline «Barrière + Torches + Bruit» & $\mathrm{Br}-\mathrm{G}$ \\
\hline «Torches + Bruit + Gardiennage» & $\mathrm{Lu} \cdot \mathrm{Br}$ \\
\hline «Barrière + Torches + Bruit + Gardiennage» & $\mathrm{Ba}-\mathrm{Lu}-\mathrm{Br}$ \\
\hline «Barrière + Bruit + Gardiennage» & $\mathrm{Lu}-\mathrm{Br}-\mathrm{G}$ \\
\hline «Barrière + Torches» & $\mathrm{Ba}-\mathrm{Lu}-\mathrm{Br}-\mathrm{G}$ \\
\hline «Torches + Gardiennage» & $\mathrm{Ba}-\mathrm{Br}-\mathrm{G}$ \\
\hline & $\mathrm{Ba}-\mathrm{Lu}$ \\
\hline & $\mathrm{Lu}-\mathrm{G}$ \\
\hline
\end{tabular}

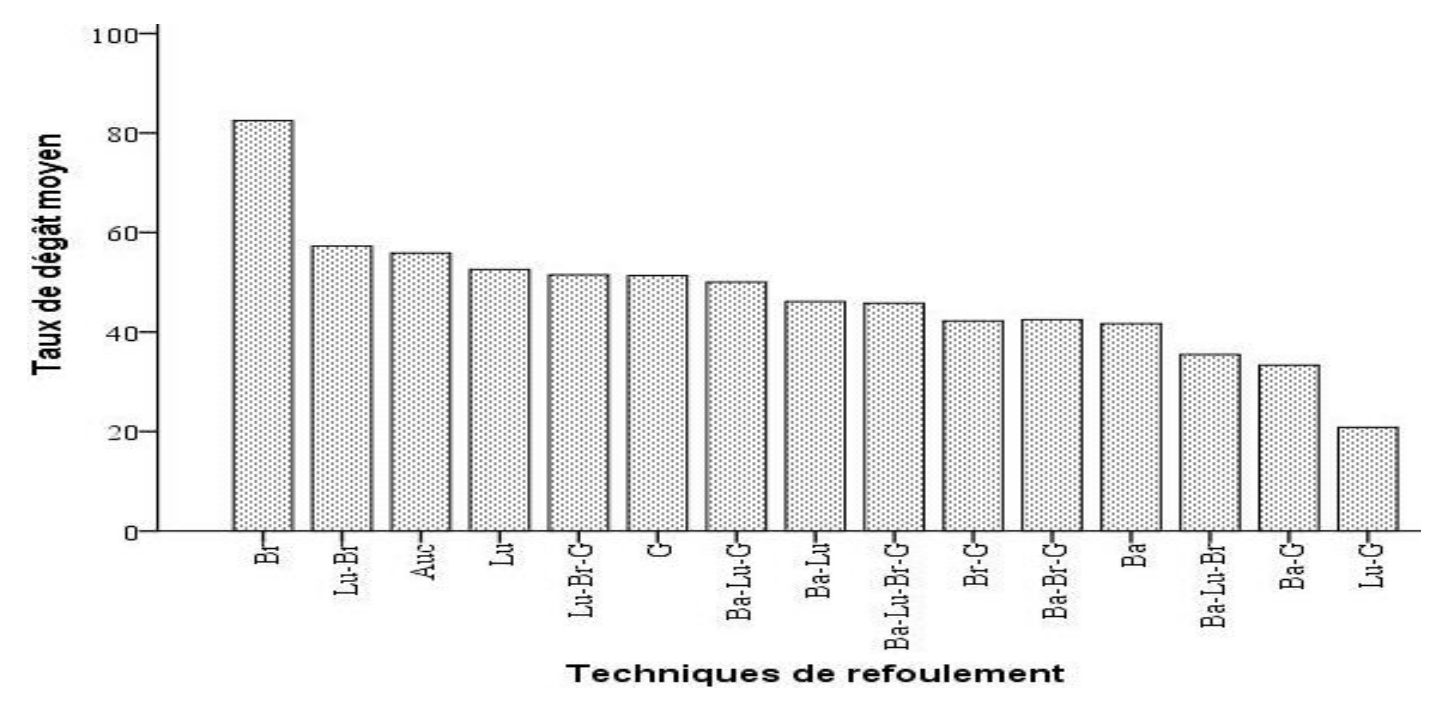

Figure 4: Taux de dégât moyen en fonction des techniques de refoulement utilisées autour de la forêt classée de Niangoloko durant les saisons agricoles de 2016 à 2018. 


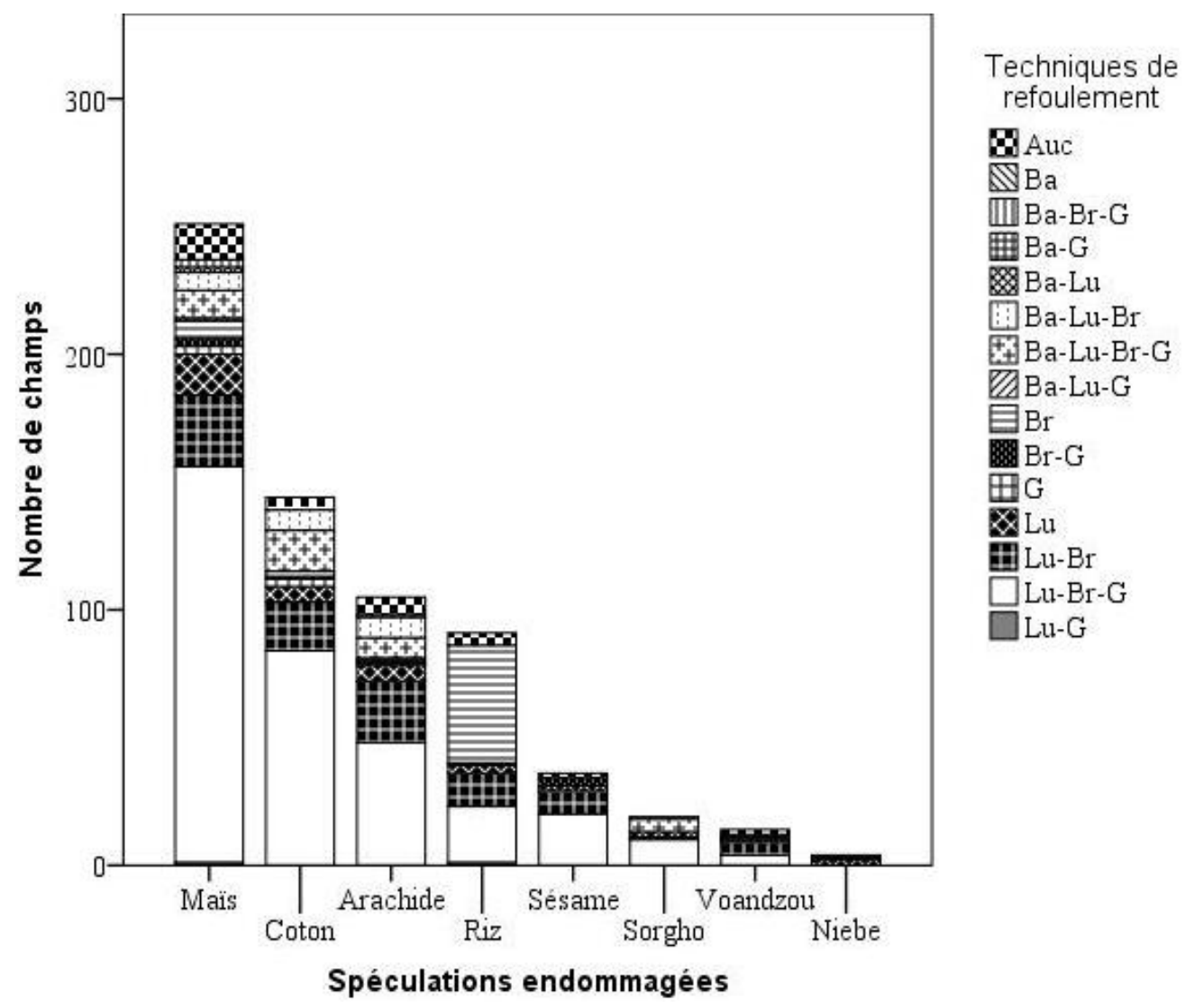

Figure 5: Nombre de champs selon la technique de refoulement utilisée en fonction du type de spéculation cultivée autour de la forêt classée de Niangoloko durant les saisons agricoles de 2016 à 2018.

Tableau 3: Résultats du test de comparaison des moyennes de taux de dégât (test de KruskalWallis) en fonction des techniques de refoulement, du type de spéculation et en fonction des années

\begin{tabular}{|c|c|}
\hline Variables & Test de K-W \\
\hline Techniques de refoulement & $\mathrm{n}=664 ; \mathrm{p}=0,000$ \\
\hline Type de spéculation & $\mathrm{n}=664 ; \mathrm{p}=0,000$ \\
\hline Années & $\mathrm{n}=664 ; \mathrm{p}=0,655$ \\
\hline
\end{tabular}


Tableau 4: Taux de dégât moyen par année en fonction des différentes techniques de refoulement autours de la FCN de 2016 à 2018. Test de comparaison des moyennes de taux de dégât (test de Kruskal-Wallis) en fonction des années selon la technique de refoulement utilisée.

\begin{tabular}{|c|c|c|c|c|c|}
\hline \multirow{2}{*}{$\begin{array}{l}\text { Technique de } \\
\text { refoulement }\end{array}$} & \multicolumn{3}{|c|}{ Taux de dégât moyen } & \multicolumn{2}{|c|}{ Test de K-W } \\
\hline & 2016 & 2017 & 2018 & $\mathrm{n}$ & Année \\
\hline $\mathrm{Ba}$ & & 50 & 33 & 2 & \\
\hline $\mathrm{Br}$ & 89 & 80 & 80 & 56 & $\mathrm{p}=0,001 *$ \\
\hline G & 57 & 44 & 57 & 7 & $\mathrm{p}=0,049^{*}$ \\
\hline $\mathrm{Lu}$ & 51 & 56 & 63 & 38 & $\mathrm{p}=0,3$ \\
\hline Auc & 55 & 59 & 55 & 36 & $\mathrm{p}=0,007 *$ \\
\hline Ba-G & 33 & & & 2 & \\
\hline $\mathrm{Br}-\mathrm{G}$ & 34 & 49 & 48 & 9 & $\mathrm{p}=0,14$ \\
\hline $\mathrm{Lu}-\mathrm{Br}$ & 59 & 56 & 57 & 99 & $\mathrm{p}=0,001^{*}$ \\
\hline $\mathrm{Ba}-\mathrm{Lu}-\mathrm{Br}$ & 27 & 38 & 38 & 23 & $\mathrm{p}=0,07$ \\
\hline Lu-Br-G & 54 & 52 & 49 & 343 & $\mathrm{p}=0,001 *$ \\
\hline Ba-Lu-Br-G & 44 & 49 & 45 & 40 & $\mathrm{p}=0,02 *$ \\
\hline $\mathrm{Ba}-\mathrm{Br}-\mathrm{G}$ & 43 & 41 & 43 & 3 & $\mathrm{p}=0,99$ \\
\hline $\mathrm{Ba}-\mathrm{Lu}$ & & 53 & 33 & 3 & \\
\hline Lu-G & & 21 & & 2 & \\
\hline Ba-Lu-G & & & 50 & 1 & \\
\hline
\end{tabular}

Tableau 5: Résultat du test de corrélation de Pearson entre le taux de dégât et le nombre d'incident.

\begin{tabular}{llcc}
\hline & \multicolumn{1}{c}{ Taux } & Nombre d'incident \\
\hline Taux & $\mathrm{R}$ & 1 & 0,123 \\
\cline { 2 - 4 } & $\mathrm{p}$ & & $0,001^{*}$ \\
\hline Nombre d'incident & $\mathrm{R}$ & 0,123 & 1 \\
\cline { 2 - 4 } & $\mathrm{p}$ & 0,001 & \\
\cline { 2 - 4 } & $\mathrm{n}$ & 664 & 664 \\
*Test significatif à $\mathrm{p}<0,01$ & $\mathrm{n}$ n: nombre de champs &
\end{tabular}


Tableau 5: Résultat du modèle linéaire généralisé (GLM) pour les trois saisons agricoles (2016 à 2018), montrant le rapport considérable entre le taux de dégât d'éléphants et son rapport avec les variables spéculations, techniques de refoulement et distances mesurées. Les variables sans effet ont été retirées du modèle.

\begin{tabular}{|c|c|c|c|c|c|}
\hline $\begin{array}{l}\text { Variables } \\
\text { Explicatives }\end{array}$ & $\begin{array}{l}\text { Corrélation } \\
\text { de dégât }\end{array}$ & Estimation & E.S & Wald & $\mathbf{p}$ \\
\hline & Constante & $-31,791$ & 43,045 & 0,545 & 0,46 \\
\hline \multirow{6}{*}{ Spéculations } & Sorgho & $-5,188$ & 23,8258 & 0,047 & 0,828 \\
\hline & Arachide & $-8,176$ & 15,514 & 0,278 & 0,598 \\
\hline & Coton & $-21,104$ & 15,832 & 1,777 & 0,183 \\
\hline & Maïs & $-15,202$ & 15,4838 & 0,964 & 0,326 \\
\hline & Riz & 20,89 & 16,5822 & 1,587 & 0,208 \\
\hline & Sésame & $-17,655$ & 17,5322 & 1,014 & 0,314 \\
\hline \multirow{8}{*}{$\begin{array}{l}\text { Techniques de } \\
\text { refoulement }\end{array}$} & $\begin{array}{l}\text { Aucune } \\
\text { technique }\end{array}$ & 12,172 & 26,7776 & 0,207 & 0,649 \\
\hline & $\mathrm{Ba}-\mathrm{Br}-\mathrm{G}$ & $-10,328$ & 25,8704 & 0,159 & 0,69 \\
\hline & $\mathrm{Ba}-\mathrm{Lu}-\mathrm{Br}$ & $-21,394$ & 15,2576 & 1,966 & 0,161 \\
\hline & Ba-Lu-Br-G & $-18,36$ & 15,2525 & 1,449 & 0,229 \\
\hline & $\mathrm{Br}$ & 7,978 & 10,7517 & 0,551 & 0,458 \\
\hline & $\mathrm{Br}-\mathrm{G}$ & 27,516 & 27,8203 & 0,978 & 0,323 \\
\hline & G & 6,568 & 18,1524 & 0,131 & 0,717 \\
\hline & $\mathrm{Lu}-\mathrm{Br}$ & 0,957 & 7,098 & 0,018 & 0,893 \\
\hline \multirow{9}{*}{ Distances } & Altitude & 0,316 & 0,1406 & 5,04 & $0,025^{*}$ \\
\hline & Champ & $-0,004$ & 0,0093 & 0,152 & 0,697 \\
\hline & Abeille & 0,002 & 0,0026 & 0,811 & 0,368 \\
\hline & Bas-fond & $-0,018$ & 0,011 & 2,634 & 0,105 \\
\hline & Forêt & $-0,001$ & 0,0027 & 0,136 & 0,712 \\
\hline & Village & $-0,003$ & 0,0038 & 0,833 & 0,362 \\
\hline & Rivière & 0,003 & 0,0025 & 1,406 & 0,236 \\
\hline & Voie & 0,004 & 0,0045 & 0,963 & 0,326 \\
\hline & (Échelle) & 610,722 & & & \\
\hline
\end{tabular}

*Significatif à $\mathrm{p}<0,05 ; \quad$ E.S: Erreur standard. 


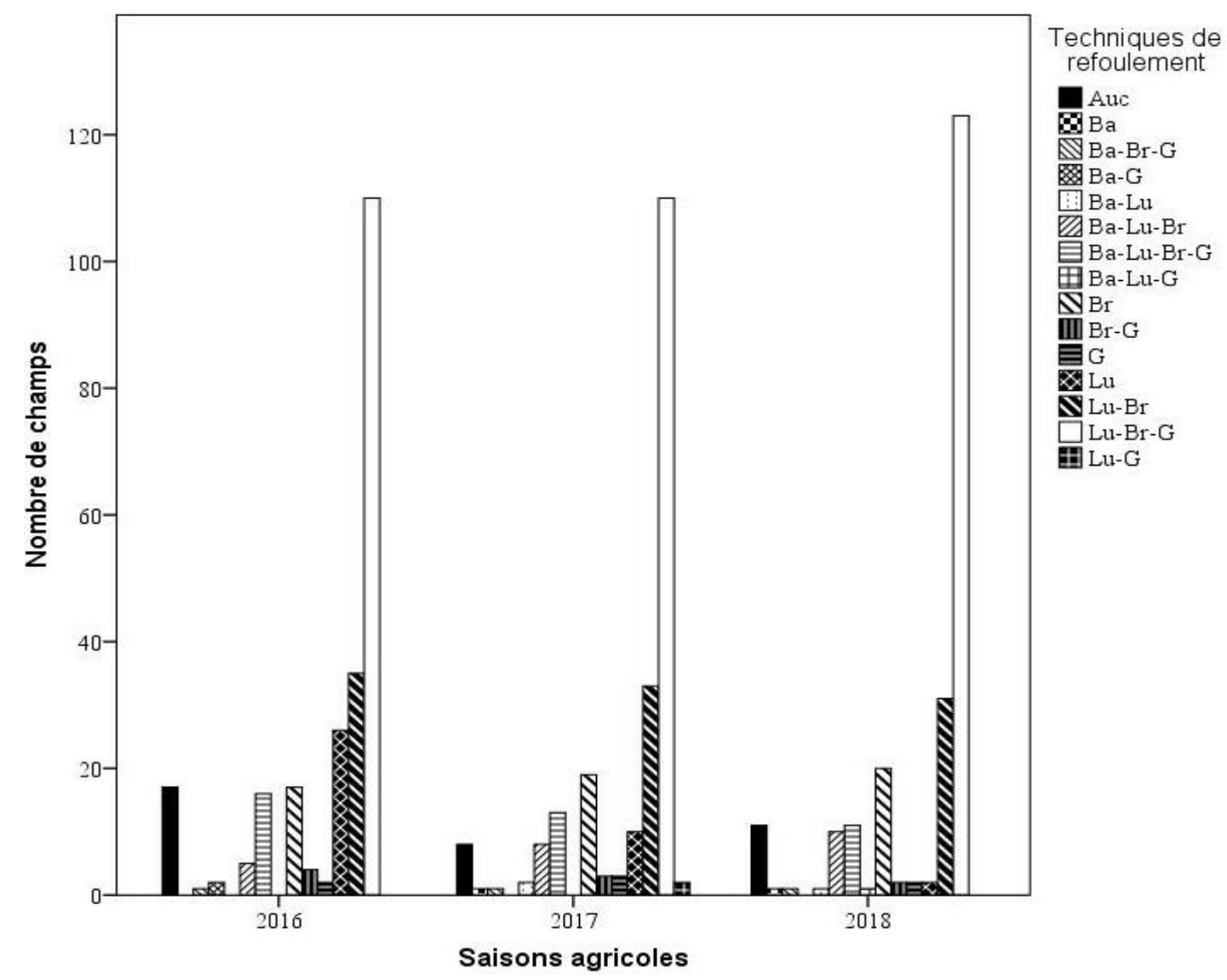

Figure 6 : Nombre de champs selon la technique de refoulement utilisée en fonction des saisons agricoles de 2016 à 2018.

\section{DISCUSSION}

Le phénomène de dégâts causés par les éléphants aux cultures est actuellement l'une des formes mondiales de conflit entre les Hommes et éléphants (Sitati et al., 2005). Autour de la FCN, la perte de $53 \%$ de la superficie cultivée serait liée à plusieurs facteurs : premièrement, à la nature des spéculations cultivées autours de la forêt car les éléphants ont une appétence pour les cultures par rapport aux plantes sauvages (Osborn, 2004). Parmi les espèces, un certain nombre attire plus l'éléphant, notamment le maïs (Chiyo et al., 2005), le riz (Davies et al., 2011), le sorgho (Hema et al., 2018). Secondairement, la proximité des champs de la limite de la forêt (Kouao et al., 2018). En effet, la FCN est une petite forêt par rapport aux autres forêts du Burkina où des CHE se déroulent (Hema et al., 2018 ; Nakandé et al.,2007). Tout le pourtour de la forêt est entièrement occupé par les champs et habitations humaines, de sorte qu'il n'y a pas de couloir réservé aux mouvements des éléphants vers les points d'eau et les forêts voisines (la Réserve Partielle de Faune de la Comoé Léraba et la forêt classée de BoulonKoflandé). Ce qui sans doute aurait déjà atténué les taux de dégâts dans la zone (Areendran et al., 2017). Enfin, l'ampleur des dégâts peut s'expliquer à travers un certain nombre de variables environnementales à savoir la superficie de la forêt, l'absence de couloir et de corridors.

Selon la nature des spéculations, autour de la FCN, le riz a été la spéculation la plus 
endommagée et la perte s'élève à $86 \%$. Davies et al. (2011) avaient fait les mêmes constats autour de district Goalpara et Sonitpur dans l'Etat d'Assam en Inde où la perte au niveau du riz s'élevait à $91,2 \%$ de la superficie totale endommagée. En effet sur le plan minéral, le riz est pauvre en sodium (Liboga et al., 2019), pourtant Rode et al. (2006) avaient constaté au Kibale National Park en Uganda que les spéculations consommées présentaient des concentrations de sodium supérieures à celles des spéculations non consommées. La consommation du riz serait donc liée à sa disponibilité (Fernando et al., 2008b ; Varma et al., 2008; Santipillai et al., 2011) et à sa distribution spatiale qui sont fortement liées aux points d'eau et aux zones inondables autour de la FCN. Plusieurs travaux ont montré que la distribution des éléphants était corrélée positivement aux point d'eau (Chamaille-Jammes et al., 2007; Tehou et al., 2019), cette corrélation était plus accentuée dans la savane (Hema et al., 2010a).

Selon l'effort de protection des cultures, la diversité des techniques (14) prouve déjà qu'un effort est fait par les producteurs vivant autour de la FCN, même si celle-ci reste insuffisant au regard des pertes dues aux éléphants qui est de 53\%. Du point de vu de l'abandon de certaines techniques après 2016, notamment Ba-G et Lu-G, les pertes moyennes de $33 \%$ et $21 \%$ ne pourraient être justifié par les pertes engendrées par leur utilisation car les techniques $\mathrm{Br}, \mathrm{G}$ et $\mathrm{Lu}$ ont été utilisées durant les trois saisons agricoles et les pertes moyennes dépassent $50 \%$ autour de la FCN. La sensibilité des producteurs pourraient être la raison qui justifie l'abandon des techniques où l'acquisition de nouvelles techniques. En effet, selon les travaux de Frank et al. (2015) sur les conflits Homme sanglier, il est ressorti que pour résoudre les conflits, les décideurs doivent aller au-delà de la gestion standard en adaptant leurs pratiques au contexte social spécifique dans lequel ils travaillent. Il est très important donc de prendre en compte le contexte social dans lequel les conflits Homme-Faune se produisent (Jepson et Canney, 2003 ; Lenin et Sukumar, 2011 ; Frank et al., 2015; deSilva, 2016). Le nombre de producteurs utilisant la technique de refoulement $\mathrm{Lu}-\mathrm{Br}-\mathrm{G}$ a augmenté de 2016 à 2018; de 110 à 123 producteurs. Selon Chiyo (2000), l'hétérogénéité des cultures et leur distribution dans l'espace sont des facteurs importants qui influencent l'efficacité de toute technique de refoulement utilisé par un producteur afin de limiter les pertes.

Les résultats du modèle GLM, montrent qu'autour de la FCN, le taux de dégât est influencé significativement par l'altitude. En effet, des études antérieures ont indiqué que la distribution des éléphants est plus liée en saisons pluvieuse aux spéculations mures (Boafo et al., 2004), telles que le maïs (Chiyo et al., 2005), le sorgho (Hema et al., 2018). Ainsi, en basse altitude, ils se déplaceraient préférentiellement en plein air dans les habitats forestiers généralement situés sur des sols bien drainés, il s'agit des sols situés à des distances intermédiaires des zones inondées (Shannon et al., 2006b). Nellemann et al. (2002) ont démontré que la rugosité du terrain est un facteur important lié à l'exploitation de l'habitat par les éléphants. Les éléphants de la FCN éviteraient les installations humaines (Ouédraogo et al., 2009).

\section{Conclusion}

Le présent travail a permis d'identifier les corrélats écologiques qui expliquent au mieux l'ampleur des dégâts dans les champs, le suivi du CHE sur trois saisons agricoles a permis de connaitre l'ampleur des dommages causés par les éléphants aux cultures et aussi il a permis de savoir que de multitudes techniques traditionnelles de refoulement des éléphants sont utilisées par les producteurs pour limiter les pertes. Le nombre de techniques varie d'une année à l'autre. Enfin, cette étude a permis de savoir que l'environnement, notamment l'altitude a un effet significatif sur l'ampleur des dégâts d'éléphant autour de la FCN. Dans la zone de la FCN, le maïs, de l'arachide, le coton et le riz 
étaient les spéculations les mieux protégées contre les raids d'éléphants, par les producteurs. Les changements de techniques seraient une bonne chose car il est connu que l'éléphant s'habitue vite à une technique de refoulement donné. Mais, pour être efficace, un changement de technique devrait être accompagné préalablement d'une évaluation de son efficacité à partir des pertes engendrées l'année précédente. Cette évaluation devrait être faite par les gestionnaires, les services techniques des forêts. Une enquête sociale liée à l'utilisation des techniques de refoulement pourrait permettre de mieux comprendre les producteurs autours de la FCN.

\section{CONFLIT D'INTERETS}

Les auteurs de cet article déclarent ne pas avoir de conflit d'intérêts.

\section{CONTRIBUTIONS DES AUTEURS}

SD a assuré la collecte de données et la rédaction du manuscrit. KI a assuré l'analyse statistique des données. EMH a assuré la planification des activités de recherche. La supervision des activités de recherche a été faite par KBG

\section{REMERCIEMENTS}

Nous remercions l'AGEREF/ CL pour le soutien logistique durant les activités de terrain.

\section{REFERENCES}

Areendran G, Raj K, Mazumdar S, Sharma A. 2017. Land use and land cover change analysis for Kosi River wildlife corridor in Terai Arc Landscape of Northern India: Implications for future management. Tropical Ecology, 58(1): 138-149. tropecol.com/pdf/open/PDF_58_1/13.

Boafo Y, Dubiure UF, Danquah, EKA, Manford M, Nandjui A, Hema EM, Barnes, RFW, Bailey B. 2004. Long-term management of crop-raiding by elephants around the Kakum Conservation Area in southern Ghana. Pachyderm, 37: 68-72. https://www.researchgate.net/publication/2 64157639.

Chamaillé-Jammes S, Valeix M, Fritz $\mathrm{H}$. 2007. Managing heterogeneity in elephant distribution: Interactions between elephant population density and surface-water availability. Journal of Applied Ecology, 44: 625-633. DOI: $10.1111 / \mathrm{j} .1365-$ 2664.2007. 01300.x

Chiyo PI. 2000. Elephant ecology and crop depredation in Kibale National Park, Uganda. M.Sc. Thesis. Makerere University. Kampala, Uganda.

Chiyo P, Cochrane EP, Naughton L, Basuta G. 2005. Temporal patterns of crop raiding by elephants: a response to changes in forage quality or crop availability. African Journal of Ecology, 43(1): 48-57. DOI:10.1111/j.1365-2028.2004.00544.x

Davies TE, Wilson S, Hazarika N, Chakrabarty J, Das D, Hodgson D. 2011. Effectiveness of intervention methods against crop-raiding elephants. Conservation Letters, 4(5): 346-54. DOI:10.1111/j.1755-263X.2011.00182.x

de Silva S. 2016. Need for longitudinal studies of Asian wildlife in the face of crises. Global Ecology and Conservation, 6: 276285. DOI: $10.1016 /$ j.gecco.2016.03.010

FAO, AFRISTAT. 2000. Réunion technique sous régionale sur les méthodes de prévisions de récoltes des cultures vivrières. Rapport de synthèse. Bamako 15-16 mai 2000. 116p. www.ofarcy.net > documentation > rapport

Fernando P, Kumar MA, Williams AC, Wikramanayake E, Aziz T, Singh S. 2008. Review of Human-Elephant Conflict Mitigation Measures Practiced in South Asia, AREAS Technical Support Document Submitted to World Bank. World Bank-WWF Alliance for Forest Conservation and Sustainable Use, Geneva, Switzerland, 45p.

Frank B, Monaco A, Bath AJ. 2015. Beyond standard wildlife management: a pathway to encompass human dimensions findings in wild boar management. European 
Journal of Wildlife Research, 61:723-730. DOI:10.1007/s10344-015-0948-y

Guinko S. 1984. La végétation de la HauteVolta. Thèse de Doctorat ès Sciences, Université de Bordeaux III. 394 p.

Hedges S, Gunaryadi D. 2010. Reducing human-elephant conflict: do chillies help deter elephants from entering crop fields? Oryx, 44: 139-146. DOI: https://doi.org/10.1017/S00306053099900 93

Hema ME, Barnes RFW, Guenda W. 2010a. Distribution of savannah elephants (Loxodonta africana africana Blumenbach 1797) within Nazinga game ranch, southern Burkina Faso. Afr. J. Ecol., 49: 141-149. DOI: $\quad 10.1111 / \mathrm{j} .1365-$ 2028.2010.01239.x

Hema EM, Sirima D, Niagabarè B, Nama N, Petrozzi F, Di Vittorio M, Barnes RFW, Guenda W, Luiselli L. 2018. Raiding or not raiding? A study of the ecological correlates of human-elephant conflict around the Nazinga Game Ranch, BURKINA FASO. Revue d'Écologie, 73(1): $1-9 . \quad$ DOI : http://hdl.handle.net/2042/64275

Hien M. 2001. Études des déplacements des éléphants, liens avec leur alimentation et la disponibilité alimentaire dans le Ranch de Gibier de Nazinga, Province du Nahouri, Burkina Faso. Thèse de Doctorat ; Université de Ouagadougou ; Burkina Faso. 136 p.

Hosmer DW, Lemeshow S. 2000. Applied Logistic Regression Analysis (2nd edn). John Wiley and Sons: New York.

INSD (Institut National de la Statistique et de la Démographie). 2017. Annuaire statistique 2016 de la région des Cascades, Burkina Faso. 202p.

Jepson P, Canney S. 2003. The state of wild Asian elephant conservation in 2003. An independent audit for Elephant Family and Conservation Direct. London, United Kingdom, $47 \mathrm{p}$.

Kouao ML, J-CK Bene, Koffi AD, Kouame BA, Kone I. 2018. Caractérisation des dégâts provoqués par la faune sauvage à la périphérie de la Forêt des Marais TanoeEhy au sud-est de la Côte d'Ivoire. Int. J. Biol. Chem. Sci., 13(1): 110-125. DOI: https://dx.doi.org/10.4314/ijbcs.v12i4.16

Lenin J, Sukumar R. 2011. Action Plan for the Mitigation of Elephant-Human Conflict in India. Asian Nature Conservation Foundation : Bangalore, India ; $122 \mathrm{p}$.

Liboga OB, Litucha BJ, Ngama BF, Balimo IF. 2019. Étude de la qualité physicochimique et technologique d'une variété locale (Kitombe) et quatre variétés améliorées du riz pluvial cultivées à Kisangani et ses environs, en R. D. Congo. J. Appl. Biosci., 139: 14158 - 14166. DOI: https://dx.doi.org/10.4314/jab.v139i1.3

Mackinnon J, Aveling C, Olivier R, Murray M, Paolini C. 2015. Éléments d'une approche stratégique de l'UE pour la conservation de la nature en Afrique Synthèse. Au-delà des éléphants. 112p.

MAHRH (Ministère de l'Agriculture, de l'Hydraulique et des Ressources Halieutiques). 2008. Resultats definitifs de l'enquête permanente agricole. Rapport d'analyse. EPA 2006-2007 (2).

Nakande A, Belem AMG, Nianogo, AJ, Jost C. 2007. Conflits hommes- éléphants dans la Réserve Partielle de Pama, Burkina Faso. Pachyderm, 42: 81-91. https://www.researchgate.net/publication/2 28680866

Ouedraogo M, Delvingt W, Doucet J-L, Vermeulen C, Bouche P. 2009. Estimation des effectifs des populations d'éléphants par la méthode d'inventaire pédestre total au Ranch de Gibier de Nazinga (Burkina Faso). Pachyderm, 45: 57-66. DOI: http://hdl.handle.net/2268/22346

Osborn FV. 2004. Seasonal influence of rainfall and crops on home-range expansion of bull elephants. Pachyderm, 35: 53-59.

Ouoba P, Lykke AM, Boussim J, Guinko S. 2006. La flore médicinale de la Forêt Classée de Niangoloko, Burkina Faso. Etudes Flor. Vég., 10: 5-16. Frankfurt / 
Ouagadougou, Oktober/octobre 2006. https://www.researchgate.net/publication/2 35675602.

Parker GE, Osborn FV, Hoare RE, Niskanen LS. 2007. Human-Elephant Conflict Mitigation: A training Course for Community-Based approaches in Africa. Trainer's manual. Elephant Pepper Development Trust, Livingstone, Zambia and IUCN/SSC AfESG, Nairobi, Kenya. 82p.

Rode KD, Chiyo, PI, Chapman CA, Mcdowell LR. 2006. Nutritional ecology of elephants in Kibale National Park, Uganda, and its relationship with crop-raiding behaviour. Journal of Tropical Ecology, 22: 441-449. DOI:

https://doi.org/10.1017/S02664674060032 33

Santiapillai C, Wijeyamohan S, Bandara G, Athurupana R., Dissayanake N, Read B. 2011. An assessment of the humanelephant conflict in Sri Lanka. Ceylon
Journal of Biological Science, 39: 21-33. DOI: $10.4038 /$ cjsbs.v39i1.2350

Sitati N, Walpole M, Leader-Williams N. 2005. Factors affecting susceptibility of farms to crop raiding by African elephants: using a predictive model to mitigate conflict. J. Appl Ecol., 42: 1175-1182. DOI: https://doi.org/10.1111/j.13652664.2005.01091.x

Tehou CA, Lougbegnon TO, Houessou LG, Mensanh GA, Sinsin B. 2019. Influence de la proximité des points d'eau sur l'intensité des dégâts des populations d'éléphants et sur les peuplements de Adansonia digitata L. et Acacia sieberiana DC., dans la Réserve de Biosphère de la Pendjari. Int. J. Biol. Chem. Sci., 13(1): 110-125. DOI: https://dx.doi.org/10.4314/ijbcs.v13i1.10

Varma S, Dand NS, Thanh TV, Sukumar R. 2008. The elephant of Cat Tien National Park, Vietnam: status and conservation of a vanishing population. Oryx, 42: 92-99. DOI: $\quad 10.1017 / \mathrm{S} 0030605308010090$ 